\title{
Comparison of Glucometry and Venous Blood Sugar Results in Diabetic Patients Undergoing Open Heart Surgery with Hemodynamic Instability
}

\section{ARTICLE INFO}

\section{Article Type}

Descriptive Study

\section{Authors}

Sajadi M. ${ }^{1} P h D$

Bijari H.* $M S c$,

Soltani M. ${ }^{2} M S c$,

Kianmehr M. ${ }^{3} P h D$

How to cite this article
Sajadi M, Bijari H, Soltani M,
Kianmehr M. Comparison of
Glucometry and Venous Blood
Sugar Results in Diabetic Patients
Undergoing Open Heart Surgery
with Hemodynamic Instability.
Horizon of Medical Sciences.
2017;23(1):1-5.

*Research Committee, Gonabad University of Medical Sciences, Gonabad, Iran

${ }^{1}$ Medical-Surgical Nursing Department, Nursing \& Midwifery Faculty, Gonabad University of Medical Sciences, Gonabad, Iran

${ }^{2}$ Anesthesiology Department, Paramedicine Faculty, Birjand University of Medical Sciences, Birjand, Iran

${ }^{3}$ Radiology Department, Medicine Faculty, Gonabad University of Medical Sciences, Gonabad, Iran

\section{Correspondence}

Address: Emergency Department, Vali-Asr Hospital, Ghaffari Street, Birjand, Iran

Phone: +98 (56) 32406200

Fax: -

hadi.bijari@gmail.com

\section{Article History}

Received: March 4, 2016

Accepted: November 12, 2016

ePublished: January 19, 2017

\section{A B S T R A C T}

Aims Blood sugar control is an important factor in mortality reduction in diabetic patients with acute and critical illness. ICU nurses have an important role in preparing and controlling the blood glucose sample. Due to the lack of evidence for appropriateness of measuring blood glucose in patients with hemodynamic instability using glucometers, the current study aimed to compare the glucometry results with venous blood sugar in diabetic patients undergoing open heart surgery with hemodynamic instability.

Instrument \& Methods In this comparative study with a time-series design, which was conducted in the heart surgery and ICU wards of Vali-Asr hospital of Birjand in 2015, 60 diabetic patients were selected based on convenience sampling method. Blood glucose samples were measured through venous blood sampling and simultaneously with glucometry method at four stages (before surgery and admission to the operating room, opening time of the thorax, admission in the ICU, and one hour after admission to the ICU). The data were analyzed with SPSS 16 software, using Pearson correlation test, repeated measures analysis and Bland and Altman's charts.

Findings There was a significant difference between glucometry blood glucose average with venous blood glucose at all four sampling occasions $(\mathrm{p}<0.001)$. Bland and Altman's chart showed difference between two methods. There was a strong correlation between two measuring methods of blood sugar $(\mathrm{p}<0.001 ; \mathrm{r}=0.94)$.

Conclusion It seems inappropriate to measure blood sugar in patients with hemodynamic instability via the glucometry method and venous blood sampling seems more reasonable.

Keywords Diabetes Mellitus; Blood Glucose; Cardiac Surgical Procedures; Hemodynamic

\section{CIT A T I O N L INKS}

[1] Non-insulin-dependent diabetes mellitus ... [2] Correlation between self-care and selfefficacy in adolescents with ... [3] Pain perception of different lancing sites during selfmonitoring of blood glucose; a comparison of fingertip with calf lancing in type ii diabetic ... [4] Effect of educational intervention on oral health self-care behaviors in diabetic ... [5] The cost of diabetes chronic complications among Iranian people with type 2 diabetes ... [6] Attitude toward preventive behavior of complications ... [7] The nursing needs special ... [8] Effect of sample storage temperature and time delay on blood gases, bicarbonate and $\mathrm{pH}$ in human arterial blood ... [9] Medical physiology ... [10] Physiological differences between interstitial glucose and blood glucose measured in human ... [11] Factors associated with prolonged mechanical ventilation following coronary artery bypass graft ... [12] A review of cardiac rehabilitation benefits on physiological aspects in patients with cardiovascular ... [13] Comparison of pleural and pericardial drainage with medial or lateral thoracostomy drain in Shariati Hospital in ... [14] Rapid changes in postprandial blood glucose produce concentration differences at finger, forearm, and thigh sampling ... [15] Negative pressure suction during blood sampling may reduce the difference in self-monitoring of blood glucose results between fingertip pricking and forearm ... [16] Accuracy of bedside capillary blood glucose measurements in critically ill ... [17] Comparison between arterial and capillary blood glucose monitoring in patients with ... [18] Bedside capillary blood glucose measurements in critically ill patients: Influence of catecholamine ... [19] Accuracy of bloodglucose measurements using glucose meters and arterial blood gas analyzers in critically ill adult ... [20] Misleading result from a capillary blood sugar sample during acute ... [21] High blood glucose level on hospital admission and poor neurological recovery after cardiac ... [22] A comparision of blood glucose level measured by ... [23] Measurement of beta tapes czech ... [24] Glucose meters: a review of technical challenges to ... [25] Comparison of blood glucose measurements using samples obtained from the forearm, finger skin puncture, and venous .. 


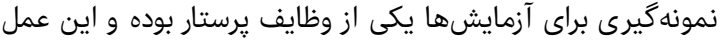

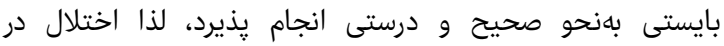

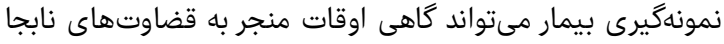

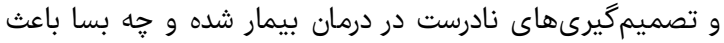

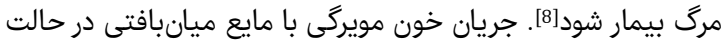

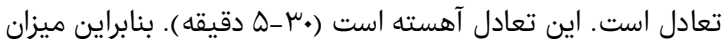

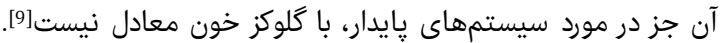

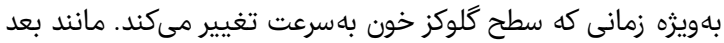

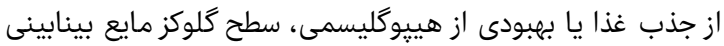

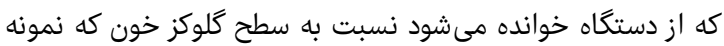

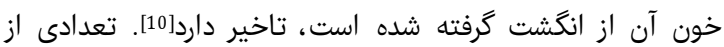

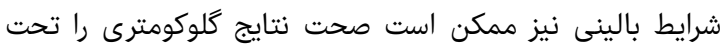

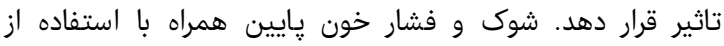

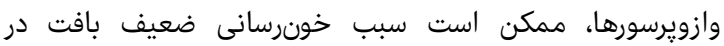

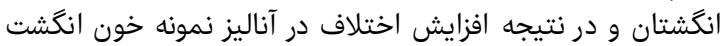

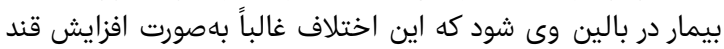

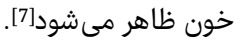

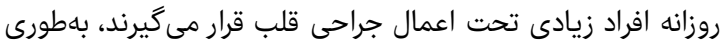

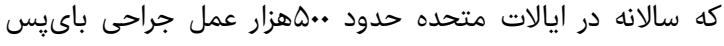

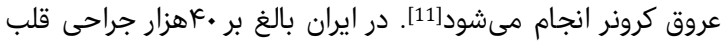

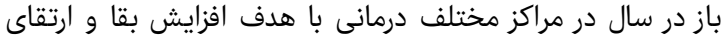

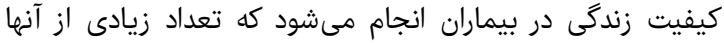

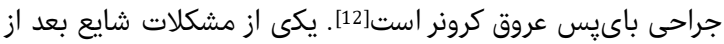

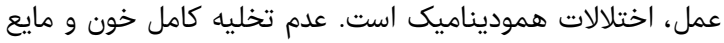

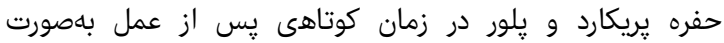

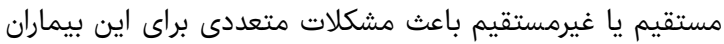

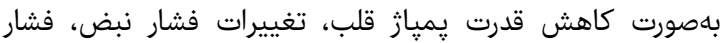

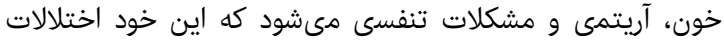
هموديناميك بعد از عمل را در يُ ديى دارد[13].

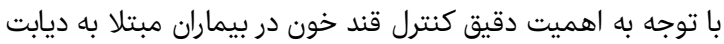

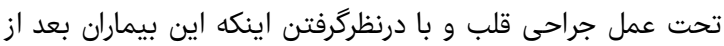

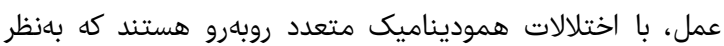

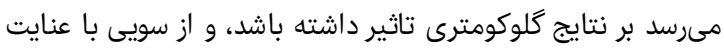

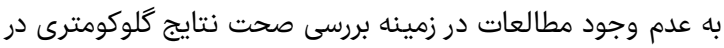

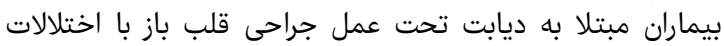

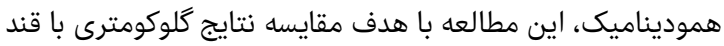

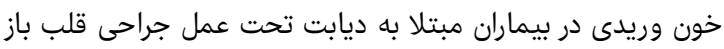
دجار اختلالات هموديناميك انجام شد.

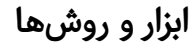

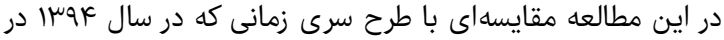

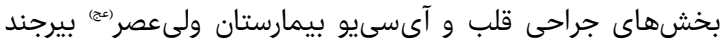

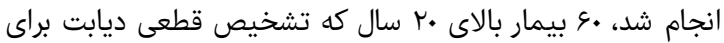

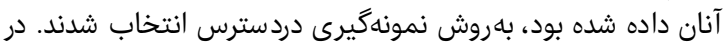

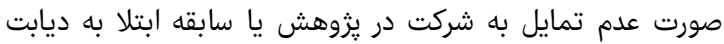

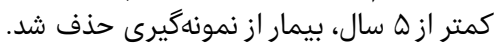

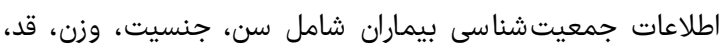

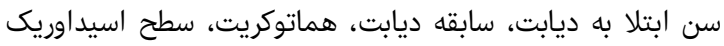

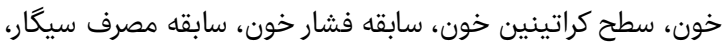

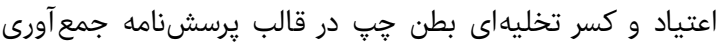

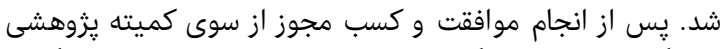
دانشكاه علوم يزشكى كناباد و كسب كد از كميته اخلاق دانشكاه و و

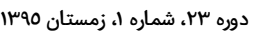

مقايسه نتايج كلوكومترى با قند خون وريدى در بيماران ديابتى تحت عمل جراحى قلب بائ باز دجار اختلالات هموديناميك ديت

موسى سجادى PhD

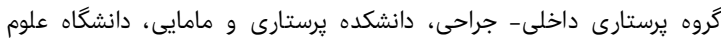
يزشكى گَناباد، كناباد، ايران

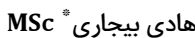

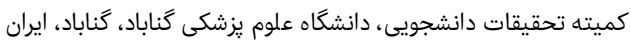

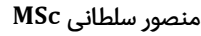
كروه هوشبرى، دانشكده يِيرايزشكى، دانشكاه علوم يزشكى بيرجند، بيرجند، ايران PhD مجتبى كيانميري

كروه راديولوزى، دانشكده يزشكى، دانشكاه علوم يزشكى گَناباد، گَناباد، ايران

קكيده

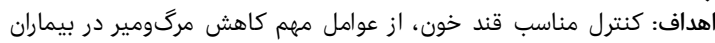

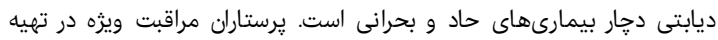

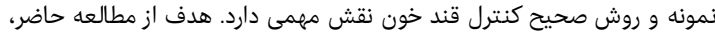

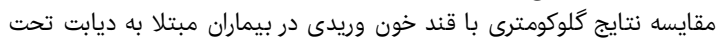

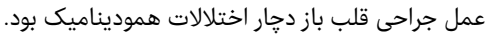

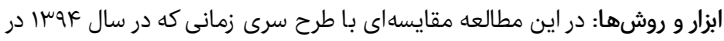

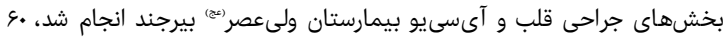

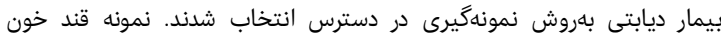

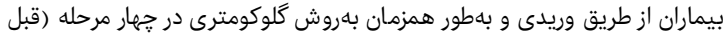

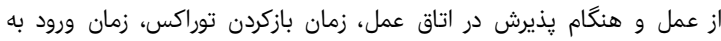

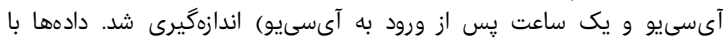

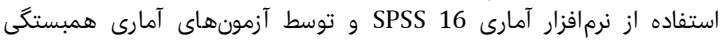

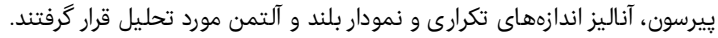

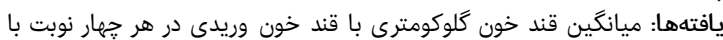

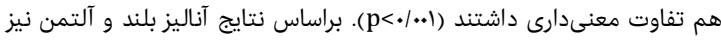

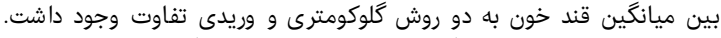

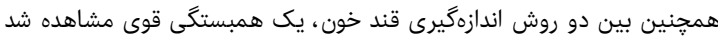

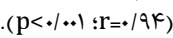

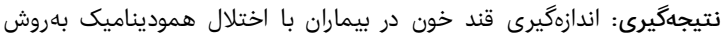

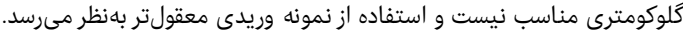

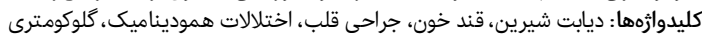

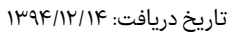

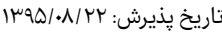

"نويسنده مسئول: hadi.bijari@gmail.com

مقدمه

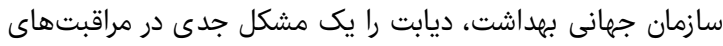

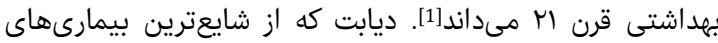

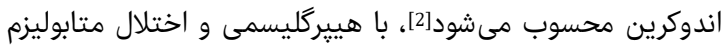

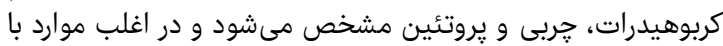

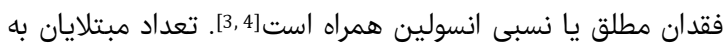

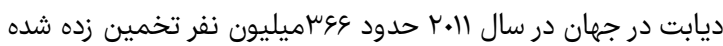

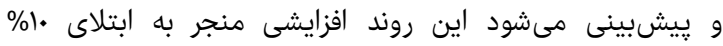

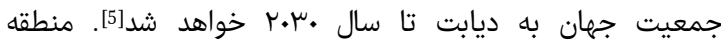

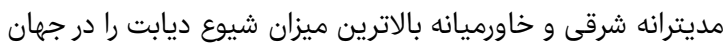

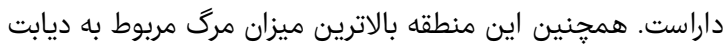

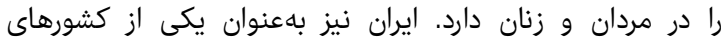

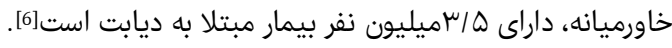

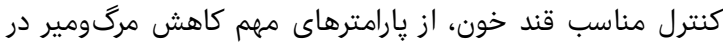

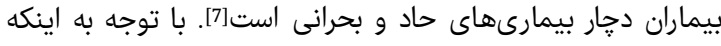




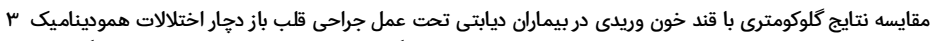

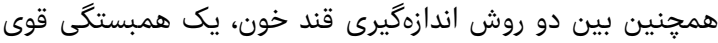

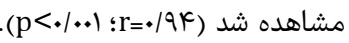

جدول r ) مقايسه ميانكَين آمارى قند خون بر حسب ميلى گرم بر دسىليتر با دو

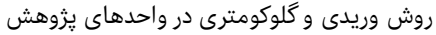

\begin{tabular}{|c|c|c|}
\hline روش وريدى & روش گلوكومترى & نوبت \\
\hline $198 / V / \pm \Delta F / 9 \Lambda$ & $r \cdot \Lambda / 9 / \pm \Delta q / / r$ & اول \\
\hline $19 \vee / / 4 \pm \Delta \mu / \wedge q$ & $M \mid Y / \cdot \wedge \pm \varepsilon \wedge / V K$ & 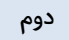 \\
\hline $19 V / / \Delta \pm \Delta F / \Delta S$ & $r \cdot \vartheta / V \Delta \pm \Delta V / F \Delta$ & سوم \\
\hline$r \cdot V / r \Delta \pm \Delta \Psi / Q r$ & $r r .1 \cdot 1 \pm 91 / .9$ & جهקارم \\
\hline
\end{tabular}

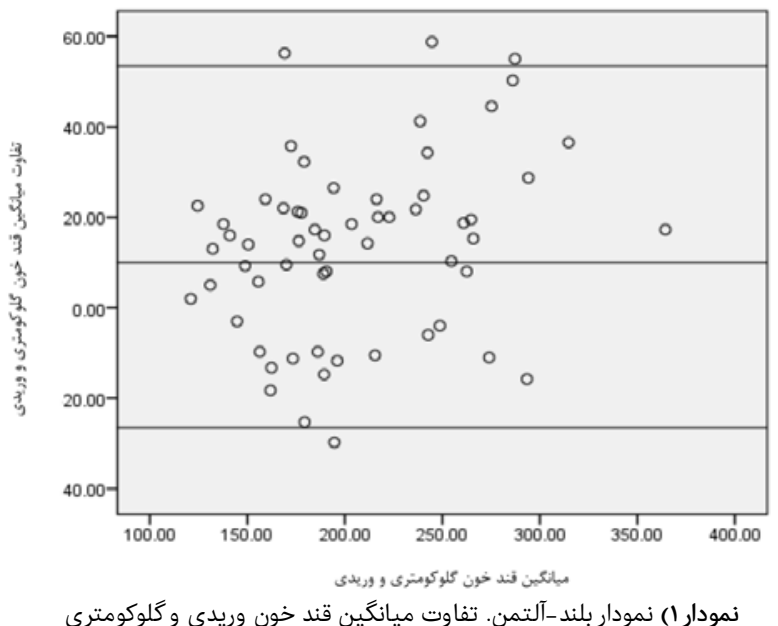

نمودار () نمودار بلند-آلتمن. تفاوت ميانكين قند خون وريدى وكلوكومترى

بحث

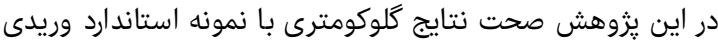
در بيماران آىسىيو جراحى قلب كه از نظر بالينى دهّار اختلالات

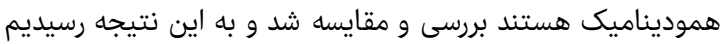

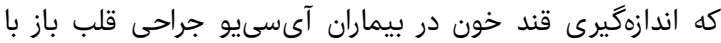

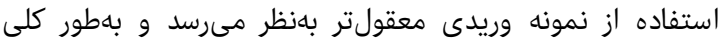

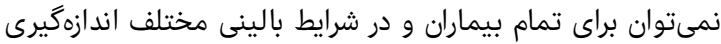

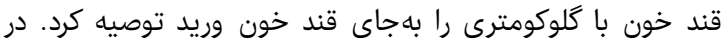

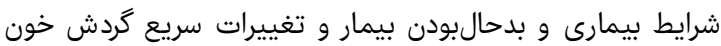

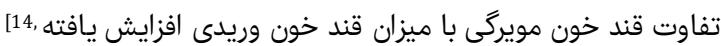

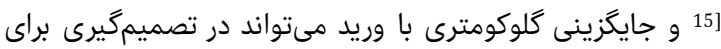

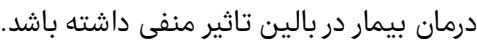

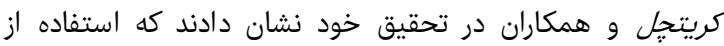

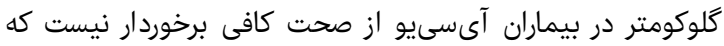

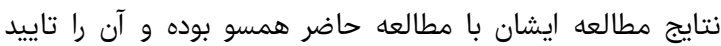

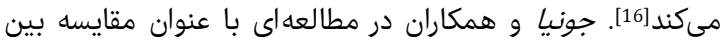

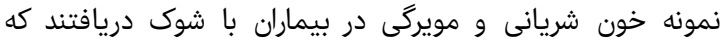

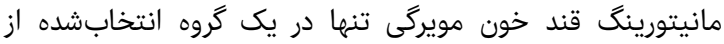

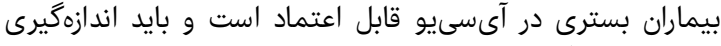

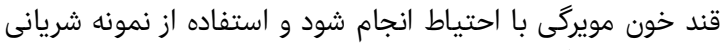

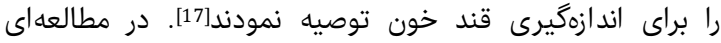

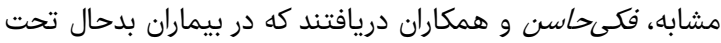

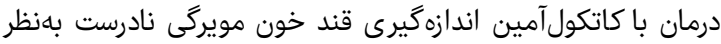

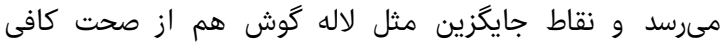

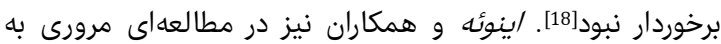

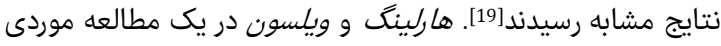

هماهنگى با محيطهاى يزوهش اقدام به نمونهيرى و انجام

مطالعه شد. - ماهن

يس از مراجعه به محيط يزوهش و و اخذ رضايت آكاهانه از بيماران،

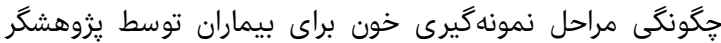

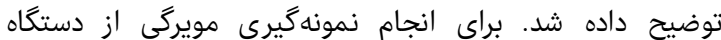
كلوكومتر Accu- Chek) مدل Active ساخت كارخانه Roche

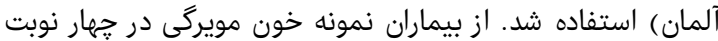

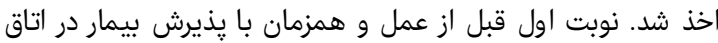

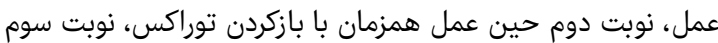

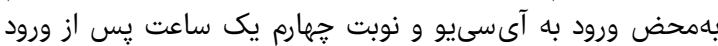

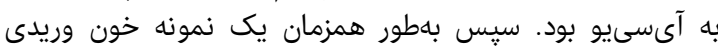

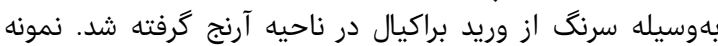

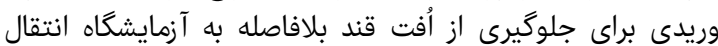

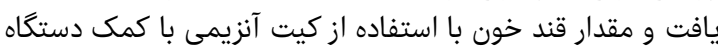

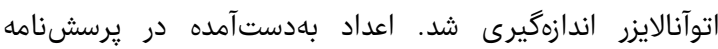

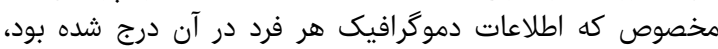

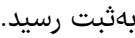
بهمنظور تجزيه و تحليل دادههاى بهدست آمدآده، ابتدا ميانگين

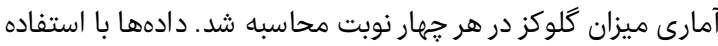

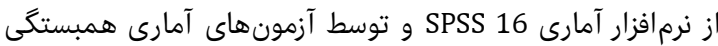

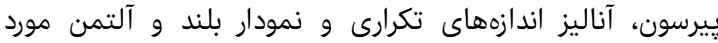

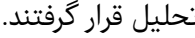

يافتهها

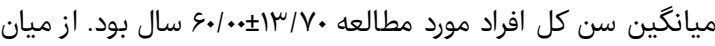

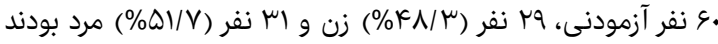

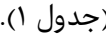

جدول () مشخصات جمعيت شناختى و بالينى در واحدهاى بزّوهش

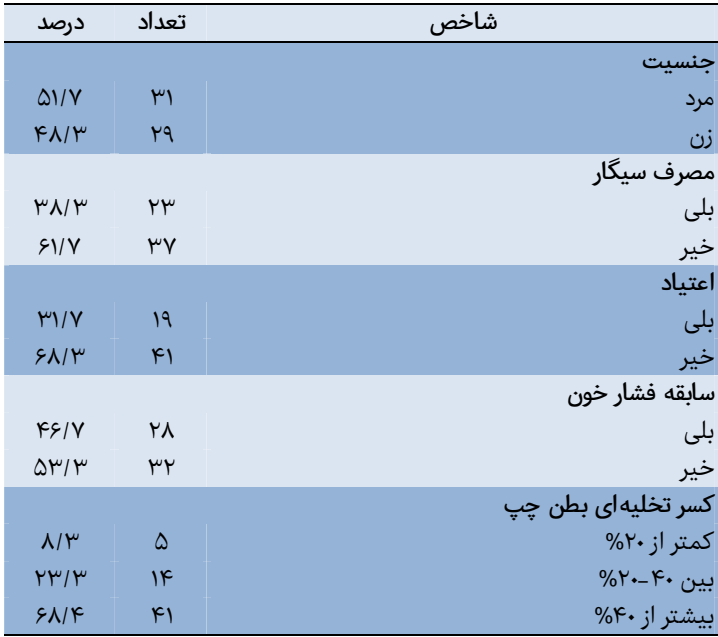

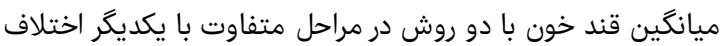

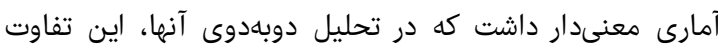

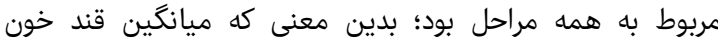

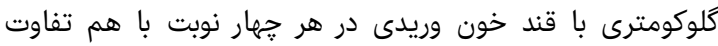

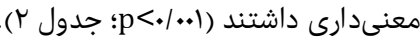

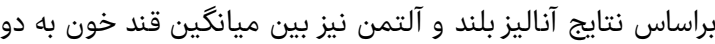
روش كلوكومترى و وريدى تفاوت وجود داشت لتيز (نمودار (). 
تعارض منافع: موردى توسط نويسندكان گزارش نشده است.

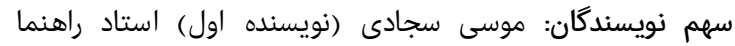

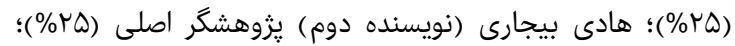

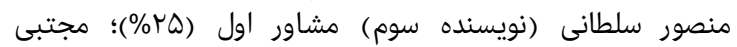

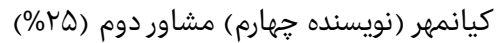

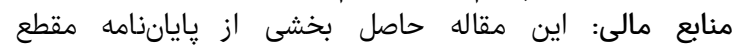

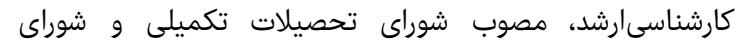

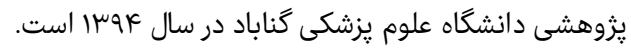

منابع

1- Harris SB, Perkins BA, Whalen-Brough E. Non-insulindependent diabetes mellitus among first nations children: New entity among first nations people of north western ontario. Can Fam Physician. 1996;42:869-76.

2- Rezasefat Balesbaneh A, Mirhaghjou SN, Jafari Asl M, Kohmanaee Sh, Kazemnejad L, Monfared A. Correlation between self-care and self-efficacy in adolescents with type 1 diabetes. Holist Nurs Midwifery. 2014;24(72):1824. [Persian]

3- Mohammadpour A, Basiri Moghadam M, Tabe'ei M. Pain perception of different lancing sites during selfmonitoring of blood glucose; a comparison of fingertip with calf lancing in type ii diabetic patients. Horizon Med Sci. 2014;20(3):185-90. [Persian]

4- Mohammadi Zeidi I, Pakpour Hajiagha A. Effect of educational intervention on oral health self-care behaviors in diabetic patients. J Isfahan Dent Sch. 2014;10(3):202-15. [Persian]

5- Farshchi A, Esteghamati A, Sari AA, Kebriaeezadeh A, Abdollahi M, Dorkoosh FA, et al. The cost of diabetes chronic complications among Iranian people with type 2 diabetes mellitus. J Diabetes Metab Disord. 2014;13(1):42.

6- Jahanfar M, Yaghmaei F, Alavi Majd H, Afkhami M, Khavari Z. Attitude toward preventive behavior of complications in type 2 diabetics. J Health Promot Manag. 2014;3(2):43-53. [Persian]

7- Cholay $\mathrm{M}$, Bronze S. The nursing needs special care. Atashzade Shuride F, Pishguyi A, Heydari M, translators. Tehran: Jamee Negar \& Salemi Publication; 2011. pp. 368-70. [Persian]

8- Mohammadhoseini E, Safavi E, Seifi S, Seifirad S, Firoozbakhsh SH, Peiman S. Effect of sample storage temperature and time delay on blood gases, bicarbonate and $\mathrm{pH}$ in human arterial blood samples. Iran Red Crescent Med J. 2015;17(3):e13577.

9- Badal Zadeh R, Ghasemi K, Rastgar Farajzadeh A. Medical physiology ganong. Tehran: Jahan Adib \& Sinai Teb Publication; 2010. pp. 527-8. [Persian]

10- Kulcu E, Tamada JA, Reach G, Potts RO, Lesho MJ. Physiological differences between interstitial glucose and blood glucose measured in human subjects. Diabetes Care. 2003;26(8):2405--9.

11- Branca P, McGaw P, Light R. Factors associated with prolonged mechanical ventilation following coronary artery bypass graft surgery. Chest. 2001;119(2):537-46.

12- Esteki Ghashghaei F, Sadeghi M, Yazdekhasti S. A review of cardiac rehabilitation benefits on physiological aspects in patients with cardiovascular disease. J Res Rehabil Sci. 2011;7(5):706-15. [Persian]

13- Sadeghpour Tabaee A, Mandegar MH, Karimi AA, Arefi S. Comparison of pleural and pericardial drainage with medial or lateral thoracostomy drain in Shariati
روى بيمار ديابتى در حال احياى قلبى- ريوى متوجه اختلاف

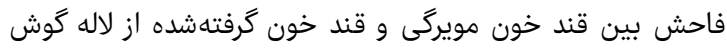

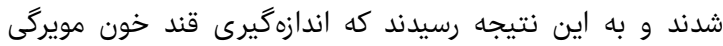

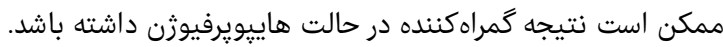

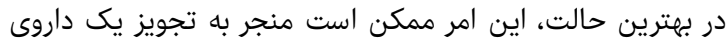

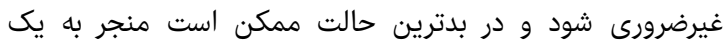

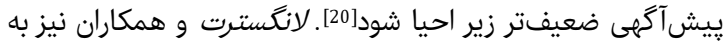

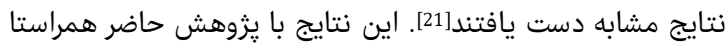
هستند.

كلاتهجارى و همكاران[22]، باستانحق و همكاران [23] و فخارى و

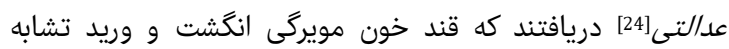

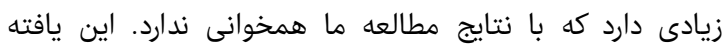

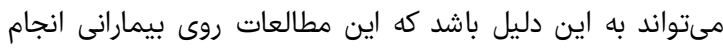

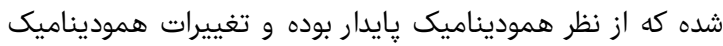

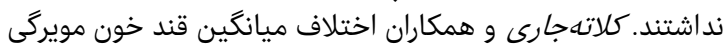

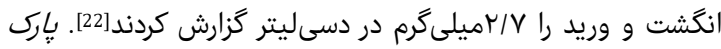

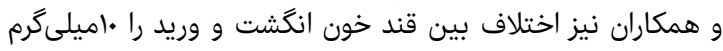

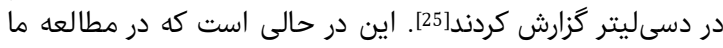

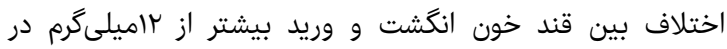

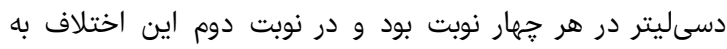
هاميلىگرم در دسى ليتر مىرسيد.

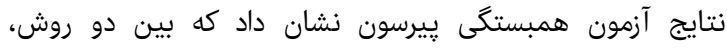

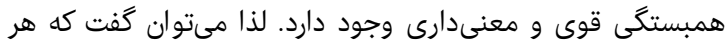

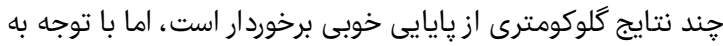

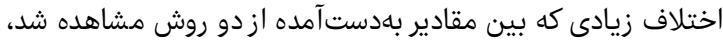

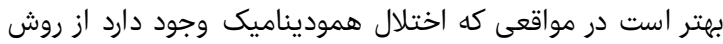

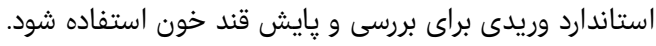

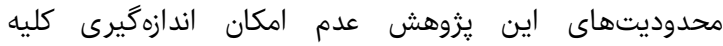

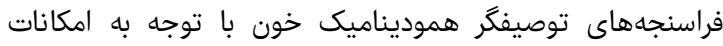

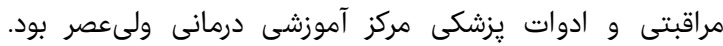

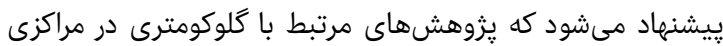

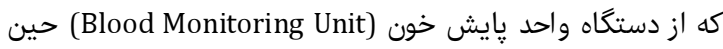

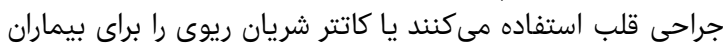
تعبيه مىنمايند، انجام يذيرد.

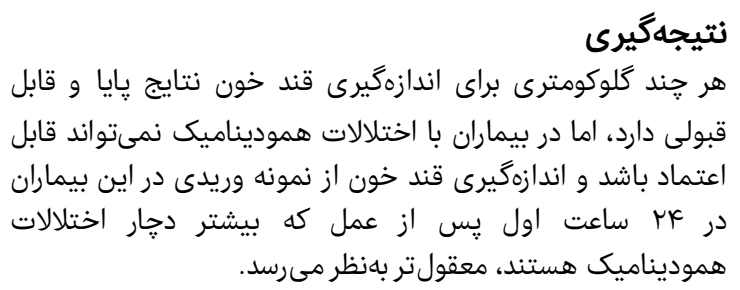
تشكر و قدردانى: يزوهش حاضر برگرفته از ياياننامه كارشناسىارشد

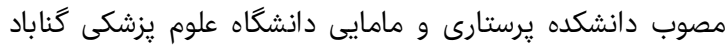

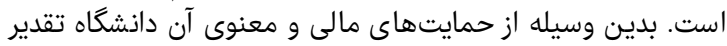

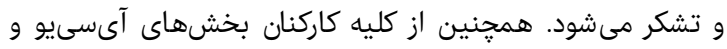

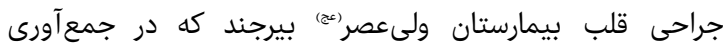

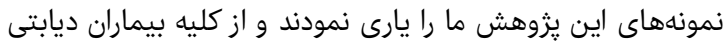

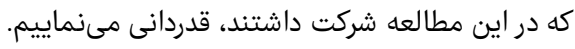

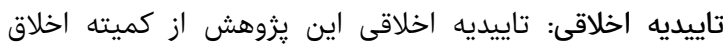

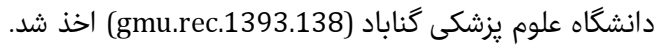


مقايسه نتايج كلوكومترى با قند خون وريدى دربيماران ديابتى تحت عمل جراحى قلب باز دجار اختلالات هموديناميك ه

blood gas analyzers in critically ill adult patients: Systematic review. Crit Care. 2013;17(2):R48.

20- Harling DW, Wilson RM. Misleading result from a capillary blood sugar sample during acute resuscitation. Resuscitation. 1995;29(2):139-41.

21- Longstreth WT Jr, Inui TS. High blood glucose level on hospital admission and poor neurological recovery after cardiac arrest. Ann Neural. 1984;15(1):59-63.

22- Ebrahimzade M. A comparision of blood glucose level measured by standard laboratory procedure with that of five glucometer devices in patients with diabetes mellitus. J Res Dev Nurs Midwifery. 2013;10(2):63-8. [Persian]

23- Bastanhagh MH, Larijani B, Khalilifard A, Hossinnejad A, Heshmat R, Khaleghian N, et al. Measurement of beta tapes czech compatible with standard. Iran J Diabetes Lipid Disord. 2003;3(1):31-4. [Persian]

24- Tonyushkina K1, Nichols JH. Glucose meters: a review of technical challenges to obtaining accurate results. J Diabetes Sci Technol. 2009;3(4):971-80.

25- Park KS, Park MS, Cha YJ, Kim WJ, Choi SS, Kim KO, et al. Comparison of blood glucose measurements using samples obtained from the forearm, finger skin puncture, and venous serum. Korean J Lab Med. 2010;30(3):26475.

Hospital in 1998-1999. Iran J Surg. 2007;15(1):1-8. [Persian]

14- Ellison JM, Stegmann JM, Colner SL, Michael RH, Sharma MK, Ervin KR, et al. Rapid changes in postprandial blood glucose produce concentration differences at finger, forearm, and thigh sampling sites. Diabetes Care. 2002;25(6):961-4.

15- Arimoto K, Sasaki H, Ogawa K, Yamasaki H, Okamoto $\mathrm{K}$, Furuta $\mathrm{H}$, et al. Negative pressure suction during blood sampling may reduce the difference in self-monitoring of blood glucose results between fingertip pricking and forearm pricking. Diabetes Care. 2004;27(6):1449-50.

16- Critchell CD1, Savarese V, Callahan A, Aboud C, Jabbour S, Marik P. Accuracy of bedside capillary blood glucose measurements in critically ill patients. Intensive Care Med. 2007;33(12):2079-84.

17- Juneja D, Pandey R, Singh O. Comparison between arterial and capillary blood glucose monitoring in patients with shock. Eur J Intern Med. 2011;22(3):241-4. 18- Fekih Hassen M, Ayed S, Gharbi R, Ben Sik Ali H, Marghli S, Elatrous S. Bedside capillary blood glucose measurements in critically ill patients: Influence of catecholamine therapy. Diabetes Res Clin Pract. 2010;87(1):87-91.

19- Inoue S, Egi M, Kotani J, Morita K. Accuracy of bloodglucose measurements using glucose meters and arterial 